\title{
SINONASAL POLYPOSIS;
}

COMPARISON OF EFFICACY OF FUNCTIONAL ENDOSCOPIC SINUS SURGERY (FESS) AND CONVENTIONAL INTRANASAL POLYPECTOMY IN SINONASAL POLYPOSIS: A RANDOMIZED CONTROLLED TRIAL.

1. FCPS

Assistant Professor

Department of Otorhinolaryngology

Kuwait Teaching Hospital Peshawar.

2. FCPS

Senior Registrar

Department of Otorhinolaryngology

Shaheed Mohtarma Benazir Bhutto Medical College,

Mirpur Azad Jammu and Kashmir.

3. MBBS

Postgraduate scholar of Public

Health, Institute of Public Health

and Social Sciences,

Khyber Medical University

Peshawar.

4. MBBS

Postgraduate Trainee,

Pakistan Institute of Medical

Sciences, Islamabad.

Correspondence

Dr. M. Salman Haider Qureshi

Postgraduate Scholar of Public Health

Khyber Medical University Peshawar.

pmc.salmanqureshi@gmail.com

Article received on:

06/11/2017

Accepted for publication:

25/04/2018

Received after proof reading:

02/10/2018
Farhan Salam 1 , Ejaz Ahmad², Muhammad Salman Haider Qureshi ${ }^{3}$, Muhammad Aslam Khan ${ }^{4}$

ABSTRACT... Introduction: Nasal polyposis (NP) is a chronic inflammatory disease involving mucous membrane in paranasal sinuses and nose. It presents as gelatinous, semitranslucent, smooth, pedunculated pear or round shaped masses comprising of inflamed mucosa that out-pouches into nose. The available surgical options are Functional Endoscopic Sinus Surgery (FESS) and Conventional Intranasal Polypectomy (CIP). The study aims to compare FESS and CIP in sinonasal polyposis patient in term of symptomatic improvement. Study Design: Randomized controlled trial. Setting: Department of head n' neck surgery and Otorhinolaryngology, Pakistan Institute of Medical Sciences, Islamabad. Period: 01-01-2015 to 20-08-2015. Methodology: A total of 84 patients presenting with nasal polyposis were enrolled. Out of these 84 patients, 42 patients were randomly selected for FESS (Group-A). Rest of the 42 patients were selected for CIP (Group-B). All the information recorded on the Performa was entered and analysed using SPSS version 17 . The entire study duration was 31 weeks. Regarding ethical consideration, the study was approved from ethics committee of hospital. Informed consent was obtained from the study participants. Moreover, confidentiality and anonymity of subjects was assured. The patients were followed-up for 2 months duration. The entire study duration was about 8 months. Results: On the basis of scoring of nasal obstruction, in FESS Group-A, 32 (76.19\%) patients had completely patent nose after two months and only $10(23.81 \%)$ patients had partially blocked nose. On the other hand in CIP Group-B, only 16 (38.10\%) patients had completely patent nose while 26 (60.90\%) patients had partially blocked nose after two months. The comparison of sense of smell between both groups also showed that the outcome of Group-A was significantly $(p-v a l u e=0.000)$ better as compared to Group-B. The complete sense of smell was found present in 37 (88.10\%) patients in group A in contrast with 19 (45.23\%) patients in group B. Conclusion: FESS seems to be a better treatment option for sinonasal polyposis than conventional intranasal polypectomy. However study with larger population size and longer follow up duration is recommended to rationalize the results.

Key words: Conventional Intranasal Polypectomy, Functional Endoscopic Sinus Surgery, Intranasal Polypectomy, Intranasal Ethmoidectomy, Nasal Polyps,.

Article Citation: Salam F, Ahmad E, Qureshi MSH, Khan MA. Sinonasal polyposis; comparison of efficacy of functional endoscopic sinus surgery (fess) and conventional intranasal polypectomy in sinonasal polyposis: a randomized controlled trial. Professional Med J 2018; 25(10):1581-1586.

DOI:10.29309/TPMJ/18.4485

\section{INTRODUCTION}

Nasal polyposis (NP) is a chronic inflammatory disease involving mucous membrane in paranasal sinuses and nose. It presents as gelatinous, semitranslucent, smooth, pedunculated pear or round shaped masses comprising of inflamed mucosa that out-pouches into nose. ${ }^{1}$ Prevalence of nasal polyposis is estimated to be between $1 \%$ and $4 \%,{ }^{2}$ with a slight male predominance ${ }^{3}$, however in few studies the rates are even up to $32 \% .{ }^{4}$
The locally acting allergic mechanisms without involvement of systemic features are thought to intervene in pathogenesis of polyp. ${ }^{5}$ Medical conditions that are most commonly related with polyps usually include bronchiectasis, cystic fibrosis and asthma. Almost $10 \%$ of total cases of NP are associated with Samnter's triad which comprises of aspirin hypersensitivity, asthma and polyposis. ${ }^{6}$ One-third cases of nasal polyposis are associated with asthma, however only in $7 \%$ of patients with athma have got polyps. ${ }^{6}$ 
Another significant cause in formation of polyps is considered to be infection. ${ }^{7}$ Studies reveal presence of olfactory alterations in $19 \%$ of general population ${ }^{8}$ in different forms including dysosmia, hyposmia and anosmia. Major causes include trauma, sinusitis, congenital abnormalities, upper airway disorders and idiopathic. ${ }^{9,10}$ Diagnosis is carried out by taking comprehensive history, conducting detailed examination, nasal endoscopy, radiography, bacteriology, pulmonary function tests and tests for aspirin sensitivity and allergy. ${ }^{11}$

The treatment options for nasal polyps include medical treatment as well as surgery. The prolong use of steroids that are taken orally is usually not recommended. Majority of nasal steroids including budesonide and fluticasone provide an effective relief and help in increasing the airflow to nose. ${ }^{12}$

Surgical modalities include intranasal ethmoidectomy, functional endoscopic sinus surgery (FESS), simple intranasal polypectomy and external ethmoidectomy. ${ }^{9}$ FESS is a technique that is minimally invasive and utilizes endoscope for drainage and improving the ventilation process apart from removal of polyp. This technique is comparatively better than other conventional modalities because it is involved not only in polyps removal rather it also helps in opening of middle meatus clefts and thus playing it's significant role in the minimizing the recurrence.

In past 25 years endoscopic sinus surgery has largely replaced older, more radical forms of surgery that are intranasal procedure. FESS is now a well-established strategy for the treatment of sinonasal polyposis which combines several techniques to open the sinuses under direct visualization. ${ }^{13,14}$ FESS is a superior and safer procedure compared to conventional intranasal polypectomy. There is also an overall decrease in complication rate and recurrence rate of nasal polyposis. ${ }^{15}$

The current study was conducted to compare Functional endoscopic sinus surgery and conventional intranasal polypectomy in sinonasl polyposis patients in term of symptomatic improvement

\section{METHODOLOGY}

A randomized controlled trial was conducted at department of head n' neck surgery and Otorhinolaryngology, Pakistan Institute of Medical Sciences, Islamabad. Total 84 patients with nasal polyposis were inducted. Patients were randomly selected on the basis of inclusion and exclusion criteria. Out of these 84 patients, 42 patients were enrolled for FESS (Group-A) while rest of the 42 patients were selected CIP (Group-B). Sample size was calculated by using $\mathrm{WHO}$ calculator with level of significance equal to 5 , power of test equal to 90 . A total of 84 patients (42 patients in each group) with nasal polyposis were enrolled.

All patients operated having sinonasal polyposis with loss of smell sense and nasal obstruction between ages 15 years to 50 years old regardless of gender were included. Patients having nasal obstruction due to other causes like deviated nasal septum, mass in nasal cavity (benign or malignant) and patients with bleeding diathesis and head injury were excluded from the study. The study was approved from ethics committee of hospital. Patients were explained about both surgical techniques and an informed consent for either procedure was taken. Moreover, confidentiality and anonymity of subjects was assured.

The socio-demographic information and past history of disease of patients was recorded. Comprehensive clinical examination performed. Hematological and biochemical test were advised as a prerequisite for General Anesthesia. Patients had routine radiographs of nose and Para nasal sinuses and computed tomography where relevant. The speculum examination of nasal cavity was performed by trainee researcher. Patency of nose was tested and graded by using Visual Analogue Scale (VAS). Olfactory threshold assessed by squeeze bottle technique by researcher and graded by 2-point scale. Both groups underwent the procedure under General Anesthesia after a senior anesthetist had 
declared the patient fit for surgery. Post-operative care was given to both the groups. Efficacy of both the interventions was assessed in term of post operative improvement in nasal obstruction and sense of smell. Each patient was followed up for two months. All the data was entered in a predesigned pro forma by trainee researcher. Bias was controlled by exclusion criteria and brief counseling of the patients. All patients were investigated and followed up by trainee researcher. For this purpose telephonic contact of patients was taken.

The data were entered and analyzed by using SPSS version 16. Descriptive statistics used to calculate for both quantitative and qualitative variables. For qualitative variables like gender, nasal obstruction and sense of smell frequencies and percentages were calculated. For quantitative variables standard deviation and mean was calculated. Chi square test was performed to determine the difference in two groups in term of symptomatic improvement and $p$ value of $<0.05$ was considered statistically significant. The entire study duration was 31 weeks.

\section{RESULTS}

After analyzing the results it was revealed that the mean age of group A, FESS group was $33.79 \pm 10.283$ years, ranging from 17 to 51 years and in group B, CIP group the mean age was almost same with a mean of 32.43 years \pm 10.687 years, having a range from 17 to 50 years. In FESS group A, there were 24 (57.14\%) males and $18(42.16 \%)$ females and in CIP group B, 26 (61.90\%) were males and 16 (38.01\%) females in our study sample. Table-1 shows the descriptive statistics for age of patients in both groups.

The narrowing of the nasal cavity caused by nasal polyps was assessed by the help of 10-point visual analogue scale (VAS) from 0 (meaning no obstruction) to 10 (worse nasal obstruction). The mean nasal obstruction VAS score in FESS group $A$, on start of study was $7.38 \pm 1.396$ and in CIP group $B$, it was $7.74 \pm 1.483$ as given in Table-II.

On the basis of this nasal obstruction VAS score $12(28.57 \%)$ patients had partially blocked nose and 30 (71.43\%) patients had completely blocked nose in both groups A and B. After two months of treatment in both groups the nasal obstruction on the basis of VAS score decreased significantly. The mean VAS score in FESS group A just remained $0.60 \pm 1.170$ after two months of treatment and in CIP group B it decreased to a mean VAS score of $2.07 \pm 1.759$, which is although significantly $(p$-value $=0.000)$ greater than the mean VAS score as depicted in Table-III.

On the basis of this nasal obstruction score the final outcome of nasal obstruction become as in FESS group A, 32 (76.19\%) patients had completely patent nose after two months and only $10(23.81 \%)$ patients had partially blocked nose. On the other hand in CIP group only 16 (38.10\%) patients had complete recovery i.e. completely patent nose but 26 (60.90\%) patients had partially blocked nose after two months.

At the start of the study all 84 patient in both groups had no sense of smell on the basis of olfactory threshold rated by 2-point scale. The post operative symptomatic improvement was found better in Functional Endoscopic Sinus Surgery group as compared to Conventional Intranasal Polypectomy. It was noted that in FESS group A, only 5 (11.90\%) patients had sense of smell absent and in $37(88.10 \%)$ patients the sense of smell was present after two months. But in CIP group B, only 19 (45.24\%) patients had symptomatic improvement and in 23 (54.76\%) patients the sense of smell was absent.

The comparison of symptomatic improvement in with regard to sense of smell and nasal obstruction was made by using chi-square test between both groups after two months and it was found that the nasal obstruction outcome was significantly $(p$-value $=0.000)$ better in FESS group A in which 32 (76.19\%) patients had completely patent nose as compared to CIP group B in which only 16 $(38.10 \%)$ patients had completely patent nose after two months as elaborated in Table-IV.

The comparison of sense of smell between both groups also showed that the outcome of FESS (group A) was significantly ( $p$-value $=0.000$ ) 
better as compared to CIP (group B). The normal sense of smell was found in $37(88.10 \%)$ patients in FESS group A in contrast with 19 (45.23\%) patients in CIP group B as elaborated in Table-V.

\begin{tabular}{|l|l|c|c|c|c|}
\hline \multicolumn{1}{|c|}{ Group } & N & Minimum & Maximum & Mean & Std. Deviation \\
\hline Group A (FESS) & 42 & 17 & 51 & 33.79 & 10.283 \\
\hline Group B (CIP) & 42 & 17 & 50 & 32.43 & 10.687 \\
\hline
\end{tabular}

Table-I. Descriptive statistics for age of patients in both groups

\begin{tabular}{|l|c|c|c|c|c|}
\hline \multicolumn{1}{|c|}{ Group } & N & Minimum & Maximum & Mean & Std. Deviation \\
\hline Group A (FESS) & 42 & 5 & 10 & 7.38 & 1.396 \\
\hline Group B (CIP) & 42 & 5 & 10 & 7.74 & 1.483 \\
\hline
\end{tabular}

\begin{tabular}{|l|c|c|c|c|c|c|}
\hline \multicolumn{1}{|c|}{ Group } & N & Minimum & Maximum & Mean & Std. Deviation & P-value \\
\hline Group A (FESS) & 42 & 0 & 4 & 0.60 & 1.170 & 0.000 \\
\hline Group B (CIP) & 42 & 0 & 5 & 2.07 & 1.759 & 0.000 \\
\hline
\end{tabular}

Table-III. Descriptive statistics for Nasal obstruction score outcome

\begin{tabular}{|c|c|c|c|c|}
\hline \multirow{2}{*}{ Nasal obstruction Outcome } & \multicolumn{2}{|c|}{ Group } & \multirow{2}{*}{ Total } & \multirow{2}{*}{ P-value } \\
\hline & Group A (FESS) & Group B (CIP) & & \\
\hline Completely Patent & 32 & 16 & 48 & \multirow{2}{*}{0.000} \\
\hline Partially Blocked & 10 & 26 & 36 & \\
\hline Total & 42 & 42 & 84 & \\
\hline
\end{tabular}

Table-IV. Comparison of nasal obstruction outcome in both groups

\begin{tabular}{|l|c|c|c|c|}
\hline \multirow{2}{*}{ Sense of Smell Outcome } & \multicolumn{2}{|c|}{ Group } & Total & P-value \\
\hline Absent & Group A (FESS) & Group B (CIP) & 28 & 0.000 \\
\hline Normal & 5 & 23 & 56 & \\
\hline Total & 37 & 19 & 84 & \\
\hline
\end{tabular}

Table-V. Comparison of sense of smell outcome in both groups

\section{DISCUSSION}

The overall prevalence of nasal polyposis is between $1 \%$ to $4 \%^{2}$, with a slight male predominance. $^{3}$ however few studies indicate rates even upto $32 \% .{ }^{4}$ The treatment aims are to significantly reduce or eliminate NP eventually causing sinus drainage improvement, relief from nasal obstruction, and restoration of taste and sense of olfaction. ${ }^{16}$

In our study, the mean age of group A, FESS group was $33.79 \pm 10.283$ years, ranging from 17 to 51 years and in group B, Conventional Intranasal Polypectomy group the mean age be almost same with a mean of 32.43 years and standard deviation of 10.687 years, having a range from 17 to 50 years. A study from Iran reported a mean age of 41 years (ranging from11-71 y) for all patients with nasal polyposis, slightly higher than what we have found in our study. In their study 65 were male and 35 were female. ${ }^{17}$

The mean nasal obstruction VAS score in our study FESS group $A$, on start of study was $7.38 \pm 1.396$ and in CIP group $B$, it was $7.74 \pm 1.483$ and 84 patients in both groups had no sense of smell on the basis of olfactory threshold rated by 2-point scale. Two months postoperatively the nasal obstruction outcome was significantly ( $p$-value $=0.000$ ) better in FESS group $A$ in which 32 (76.19\%) patients had completely patent nose as compared to CIP group B in which only 16 (38.10\%) patients had completely patent nose and showed that the outcome of FESS group A was significantly ( $p$-value $=0.000$ ) better as compared to CIP group B at the time of two month follow up. Damm et al observed sinonasal polyposis treated by FESS, nasal obstruction improved in $84 \%$ patients. ${ }^{18}$ These results are very close to our study regarding nasal obstruction in 
NP patients.

A local study showed that surgery has very encouraging outcome. Even at first visit after surgery, $90 \%$ patients were symptoms free. Surgical options for ethmoidal nasal polyp include intranasal polypectomy with intranasal ethmoidectomy, external ethmoidectomy: Caldwell lucs approach and FESS using Wigand and Stammberger technique. Minimal invasive surgery i.e. using FESS technique is gaining popularity nowadays. ${ }^{19}$

Regarding the sense of smell we found that complete sense of smell recovered in 37 $(88.10 \%)$ patients in FESS group A in contrast with 19 (45.23\%) patients in CIP group B, which shows that sense of smell markedly improved in patients who were operated by FESS. In view of these findings, results of our study regarding symptomatic improvement of patients are within the expected range. Olfactory dysfunction affects $65 \%$ to $80 \%$ of total patients with CRS. ${ }^{20}$

Another study conducted by Pade et al. in which he inducted 206 patients presenting with impairment of sense of olfaction who opted for elected ESS, revealed that the symptoms of $23 \%$ improved, $9 \%$ got worsened and $68 \%$ remained same with no obvious change. ${ }^{21}$ Soler et al., in 2010, assessed the overall impact of different histologic markers on the outcome of olfaction following ESS. ${ }^{22}$ It was revealed that impairment of olfaction is associated with the eosinophilia of tissue and thickening of basement membrane.

These variations of results in literature are probably due to different parameters to assess the nasal obstruction and sense of smell and variation in demographics of study participants. Small sample size, limited study period and short follow-up plan are some of the limitations of our study. Larger country-wide studies are required to generalize the results.

\section{CONCLUSION}

FESS has got more advantages including better visualization, preservation of normal mucosa of nasal cavity and better recovery. Keeping these advantages in views, FESS is better treatment option for sinonasal polyposis than CIP in term of improvement of sense of olfaction and nasal obstruction. However for the purpose of generalizing the results, study with larger sample size and prolong follow up is recommended.

Copyright@ 25 Apr, 2018.

\section{REFERENCES}

1. Bachert C, Wagenmann M, Rudack C, Höpken K, Hiltebrandt M, Wang D, Cauwenberge P. The role of cytokines in infectious sinusitis and nasal polyposis. Allergy. 1998 Jan 1; 53(1):2-13.

2. Small CB, Stryszak P, Danzig M, Damiano A. Onset of symptomatic effect of mometasone furoate nasal spray in the treatment of nasal polyposis. Journal of Allergy and Clinical Immunology. 2008 Apr 30; 121(4):928-32.

3. Dalziel K, Stein K, Round A, Garside R, Royle P. Systematic review of endoscopic sinus surgery for nasal polyps.

4. Larsen PL, Tos M. Origin of nasal polyps: an endoscopic autopsy study. The Laryngoscope. 2004 Apr 1; 114(4):710-9..

5. Bachert C, Gevaert P, Holtappels G, Johansson SG, Van Cauwenberge $P$. Total and specific IgE in nasal polyps is related to local eosinophilic inflammation. Journal of allergy and clinical immunology. $2001 \mathrm{Apr}$ 30; 107(4):607-14.

6. Newton JR, Ah-See KW. A review of nasal polyposis. Therapeutics and clinical risk management. 2008 Apr; 4(2):507.

7. Xu G, Xia JH, Zhou H, Yu CZ, Zhang Y, Zuo KJ, Shi JB, $\mathrm{Li}$ HB. Interleukin $\square 6$ is essential for Staphylococcal exotoxin $B \square$ induced $T$ regulatory cell insufficiency in nasal polyps. Clinical \& Experimental Allergy. 2009 Jun $1 ; 39(6): 829-37$.

8. Dawes P, Bates G, Watson D, Lewis D, Lowe D, DRAKE LEE AB. The role of bacterial infection of the maxillary sinus in nasal polyps. Clinical Otolaryngology \& Allied Sciences. 1989 Oct 1; 14(5):447-50.

9. Ricchetti A, Landis BN, Maffioli A, Giger R, Zeng C, Lacroix JS. Effect of anti-fungal nasal lavage with amphotericin B on nasal polyposis. The Journal of Laryngology \& Otology. 2002 Apr 1; 116(04):261-3.

10. Osguthorpe JD, Hadley JA. Rhinosinusitis: Current concepts in evaluation and management. Medical Clinics of North America. 1999 Jan 1; 83(1):27-41. 
11. Razmpa E, Khajavi M, Kordbacheh MH. The prevalence of fungal infections in nasal polyposis. Acta Medica Iranica. 2007; 45(1):45-50.

12. Pawliczak R, Lewandowska-Polak A, Kowalski ML. Pathogenesis of nasal polyps: An update. Current allergy and asthma reports. 2005 Nov 1; 5(6):463-71.

13. Singh R, Hazarika P, Nayak DR, Balakrishnan R, Gangwar $\mathrm{N}$, Hazarika M. A comparison of microdebrider assisted endoscopic sinus surgery and conventional endoscopic sinus surgery for nasal polypi. Indian Journal of Otolaryngology and Head \& Neck Surgery. 2013 Jul 1; 65(3):193-6.

14. Chester AC, Sindwani R, Smith TL, Bhattacharyya N. Fatigue Improvement Following Endoscopic Sinus Surgery: A Systematic Review and Meta $\square$ Analysis. The Laryngoscope. 2008 Apr 1; 118(4):730-9.

15. Iqbal J, Raza SN, Naqvi NU, Azeem AQ, Raahat ZM. Endoscopic Sinus Surgery (ESS) verses Conventional Intranasal Polypectomy for Ethmoidal Nasal Polyposis. Pak J Otolaryngol. 2007; 23: 3.

16. Drake-Lee AB. Nasal polyps. Hospital medicine (London, England: 1998). 2004 May; 65(5):264-7.
17. Benoliel P. [Treatment of sino-nasal polyposis by Candida albicans immunotherapy: apropos of $\mathbf{4}$ cases]. Allergie et immunologie. $2001 \mathrm{Dec}$; 33(10):38894.

18. Damm M, Quante G, Jungehuelsing M, Stennert E. Impact of functional endoscopic sinus surgery on symptoms and quality of life in chronic rhinosinusitis. The Laryngoscope. 2002 Feb 1; 112(2):310-5.

19. Meltzer EO, Hamilos DL, Hadley JA, Lanza DC, Marple BF, Nicklas RA, Bachert C, Baraniuk J, Baroody FM, Benninger MS, Brook I. Rhinosinusitis: establishing definitions for clinical research and patient care. Journal of Allergy and Clinical Immunology. 2004 Dec $31 ; 114(6): 155-212$.

20. Litvack JR, Mace JC, Smith TL. Olfactory function and disease severity in chronic rhinosinusitis. American journal of rhinology \& allergy. 2009; 23(2):139.

21. Pade J, Hummel T. Olfactory function following nasal surgery. The Laryngoscope. 2008 Jul 1; 118(7):1260-4.

22. Soler ZM, Sauer DA, Mace JC, Smith TL. Ethmoid histopathology does not predict olfactory outcomes after endoscopic sinus surgery. American journal of rhinology \& allergy. 2010 Jul; 24(4):281.

\section{AUTHORSHIP AND CONTRIBUTION DECLARATION}

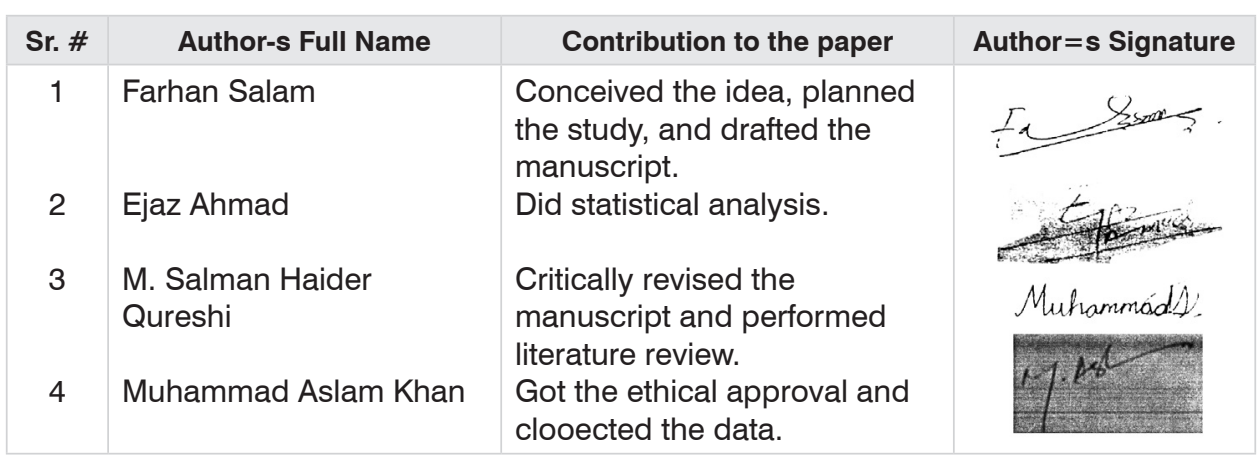

